DOCTRINA

\title{
¿Publicar y patentar? La excepción de divulgación inocua como incentivo a la revelación temprana de los resultados de investigación en la academia
}

\author{
Publish and patent? Exceptions to lack of novelty as incentives \\ for the early disclosure of research results in the academy
}

\author{
Sulan Wong (iD y Michelle Salazar Mackenna \\ Universidad de La Frontera, Chile
}

\begin{abstract}
RESUMEN Debido al requisito de novedad en la solicitud de una patente, se impone a la ciencia académica - de manera contraria al ethos mertoniano- la lógica de "patentar y luego publicar». Mediante la «excepción de divulgación inocua», figura jurídica que permite proteger temporalmente la novedad de la invención, a pesar de una revelación previa a la solicitud de una patente, podría atenuarse esta dinámica. En este trabajo, se estudia la figura en los sistemas de patentes chileno, estadounidense y español. Se concluye que la excepción es de difícil aprovechamiento porque, primero, existe una falta de armonización internacional; y, segundo, el investigador debe conocer con antelación a la publicación si un resultado es patentable.
\end{abstract}

PALABRAS CLAVE Patente, novedad, divulgación inocua, periodo de gracia.

ABSTRACT Due to the requirement of novelty in the application for a patent, the "patent, then publish" logic - contrary to the Mertonian ethos - puts pressure on academic science. By means of the "grace period", a legal institution that allows the novelty of the invention to be temporarily protected despite a disclosure made in advance of a patent application, this dynamic could be mitigated. In this work, this exception is studied in the Chilean, US and Spanish patent systems. We conclude that the advantages granted by this exception are difficult to exploit, because, firstly, there is a lack of international harmonization, and, secondly, the researcher must know, before the publication of his manuscript, if his research results are patentable.

KEYWORDS Patent, novelty, exceptions to lack of novelty, grace period. 


\section{Introducción}

La discusión histórica de la propiedad intelectual en general, y la patente en particular, ha pivotado alrededor del tema de los incentivos. ${ }^{1}$ La corriente dominante, la proteccionista, concibe a la patente como el motor que incentiva la creación tecnocientífica y la revelación del conocimiento. Aparte, se ubica una corriente minoritaria, dividida entre el abolicionismo, que sostiene que las patentes no son ni han sido siempre un incentivo a la creación, y la intermedia, que reconoce el rol de la patente como incentivo a la creación y el progreso, pero que considera la necesidad de limitar el alcance del ius prohibendi del titular de la patente (Boldrin y Levine, 2008; Boyle, 2007; Burk y Lemley, 2009b; Machlup y Penrose, 1950; Pretorius, 2002; Smiers, 2006; Stallman, 2004; Stiglitz, 2007).

Otra perspectiva de los incentivos viene dada por una suerte de quid pro quo, pues se entiende que, con el objetivo de fomentar el progreso de la ciencia y de la tecnología, el Estado incentiva la revelación del conocimiento mediante el monopolio temporal sobre la invención, reconociendo la necesidad de incorporarlo en el dominio público, aunque la sociedad deba esperar (Drahos, 2010). Quien crea una invención, a diferencia de quien crea una obra literaria, artística o científica, tiene que satisfacer los tres requisitos que conforman el llamado estándar de patentabilidad: novedad, nivel inventivo y utilidad. ${ }^{2}$ En este trabajo nos concentraremos en el primero de ellos, la novedad, el requisito que exige que la invención debe ser nueva, es decir, que no se encuentre incorporada en el estado de la técnica al momento de solicitar la patente.

Para entender el problema que el requisito de novedad impone a ciencia académica, debemos comenzar explicado el ethos de la ciencia. Según Merton (1977: 344), este:

alude a un complejo emocionalmente teñido de reglas, prescripciones, costumbres, creencias, valores y presuposiciones que se consideran obligatorios para el científico. Algunos elementos de este complejo pueden ser metodológicamente deseables, pero la observancia de las reglas no está dictada solamente por consideraciones metodológicas. Este ethos, como los códigos sociales en general, se sustenta en los sentimientos de aquéllos a quienes se aplica. Frenan transgresiones, las prohibiciones internalizadas y las reacciones emocionales reprobatorias movilizadas por los defensores del ethos.

1. Desde la Conferencia de Viena de 1873, que diez años más tarde dio origen a la Convención de París, se establece un giro en los argumentos para justificar el sistema de patentes, de uno filosófico a uno económico de corte utilitarista. Véase Patel (1974).

2. Este estándar de patentabilidad se ha sido recogido en el artículo 27.1 del Acuerdo sobre los Aspectos de Propiedad Intelectual relacionados con el Comercio (ADPIC). Se considera que una invención: 1) es nueva si el problema que se dice resolver no forma parte del estado de la técnica; 2) cumple con el requisito de nivel inventivo si dicha solución técnica no resulta obvia para experto en la materia técnica; y 3) tiene utilidad o aplicación industrial si es potencialmente replicable. 
El comunitarismo, ${ }^{3}$ en el sentido no técnico y extendido de propiedad común de bienes, es uno de los elementos del ethos científico. Los hallazgos de la ciencia son el resultado de la colaboración social y, por tanto, son entregados a la comunidad científica; constituyen una herencia común en la cual el derecho del "productor» individual se encuentra limitado. Los derechos de propiedad en la ciencia se reducen a un mínimo por el código de ética científica.

La lógica "patentar y luego publicar», incentivada por el sistema de patente, es contraria al ethos científico, precisamente porque estimula comportamientos como el secretismo (Wong, Ramos Toledano y Rojas-Mora, 2018), en lugar de colaborar con la divulgación temprana de los resultados de investigación. En la praxis, todo ello significa que quienes hacen investigación científica, deben abstenerse de comunicar los resultados de investigación antes de solicitar una patente. Aún más, como lo ha advertido Ziman (2003: 121):

El secreto en la ciencia es una forma de «contaminación epistémica» a la que está expuesta toda la ciencia posacadémica. No es sólo un signo de un gran cambio en la organización social de la ciencia. Significa también el aumento de la subordinación a intereses corporativos y políticos [...] que no dan demasiado valor a la producción del conocimiento por el beneficio que pueda suponer a la sociedad. Y, sin embargo, a largo plazo, es el carácter abierto de la ciencia académica, su respeto a la norma del comunitarismo y su fundamentación en la observancia empírica, lo que - para bien o para mal- constituye la mejor garantía de su fiabilidad práctica.

Ahora, ¿es posible, como se planteó Bagley (2006), mitigar la tensión entre el ethos mertoniano y la patente, invirtiendo la lógica "patentar y luego publicar»? Algunos países han incorporado en sus legislaciones instituciones en materia de patente que constituyen excepciones al requerimiento de novedad, estableciendo que, si la divulgación de la invención se produce en determinados supuestos, no pasará a formar parte del estado de la técnica y, en consecuencia, no se verá afectado el derecho a solicitar la patente por falta de novedad. ${ }^{4}$ En este sentido, nuestro objetivo de trabajo es dual, pues nos interesa saber cuáles son los efectos de la excepción de divulgación inocua tanto a nivel nacional como internacional. Para este último objetivo, nuestro criterio para la selección de las legislaciones objeto de estudio que compararemos con el sistema chileno se ha basado en los principales destinos a los que recurren los investigadores chilenos para solicitar patentes. Según estadísticas recientes del Instituto

3. Merton denominó originalmente a este imperativo como communism, traducido al español como "comunismo». No obstante, y para evitar la carga ideológica del término, preferimos usar comunitarismo, que ha sido la traducción usada en la obra de Ziman (2003).

4. Véase el reporte SCP/12/3 Rev.2 de la OMPI, actualizado en abril de 2020, disponible en https://bit. ly/32A2Djn. 
Nacional de Propiedad Industrial de Chile (INAPI), ${ }^{5}$ Estados Unidos es el primer país que recibe solicitudes de patentes de investigadores chilenos, seguido de la Oficina Europea de Patentes; de ésta seleccionamos a España por cercanía idiomática.

Para alcanzar nuestros objetivos, hemos estructurado el trabajo de la siguiente manera: en la primera sección, analizaremos el requisito de novedad, al ser el que resulta afectado por la divulgación inocua. En la segunda parte, estudiaremos la divulgación inocua de la invención, excepción legal de interés fundamental para la ciencia académica. En tercer lugar, en un ejercicio de derecho comparado, analizaremos la configuración de la excepción de divulgación inocua en la legislación chilena, la española y la estadounidense. Cerraremos este trabajo con unas breves conclusiones acerca de cómo la falta de armonización con respecto de la figura de la excepción de divulgación inocua podría resultar paradójica al reducir las posibilidades de publicar antes de patentar.

\section{Novedad y estado de la técnica}

De los tres elementos que conforman el llamado estándar de patentabilidad, la novedad es considerada como una condición fundamental e indiscutida que implica dos propósitos: evitar el doble patentamiento de productos o procedimientos idénticos y la extracción del dominio público del conocimiento que pertenece a la sociedad. ${ }^{6}$

La novedad debe analizarse respecto del llamado «estado de la técnica». Según Botana Agra (2013: 120-125), existe un conjunto de elementos que pueden colaborar en su delimitación: el contenido, la accesibilidad al público y el momento relevante. En cuanto al contenido, se considera que el estado de la técnica está conformado por «todo» lo que haya sido puesto a disposición del público, de forma escrita, oral o

5. «Estadísticas: Patentes», Instituto Nacional de Propiedad Intelectual, disponible en https://bit. ly/31T 7 lbr.

6. Un trabajo por el Comité de Patente de la Organización Mundial de Propiedad Intelectual ha puesto de manifiesto que, de la comparación entre las prácticas y las leyes nacionales de diferentes países sobre el concepto de novedad, se puede concluir que existen al menos tres modelos conceptuales diferentes: el primero, el riguroso, que considera que si la invención reivindicada se ha divulgado explícita o inherentemente en una solicitud anterior, dicha solicitud revoca la patentabilidad de la invención reivindicada; el segundo, el amplio, que entiende que aunque la invención reivindicada — de manera explícita o inherente - no se haya divulgado plenamente en una solicitud anterior, esta última destruye la novedad de la invención reivindicada si las diferencias entre ambas son menores; y el tercero, que al vincular la novedad a la actividad inventiva, establece que la solicitud anterior de una patente anula la patentabilidad del problema de la técnica que se resuelve, si esta última carece de novedad o de actividad inventiva en relación con la referida solicitud. «"Ampliación” del concepto de novedad: Estudio inicial sobre la novedad y los efectos en el estado de la técnica de determinadas solicitudes en virtud del proyecto de artículo 8.2) del SPLT», Organización Mundial de Propiedad Intelectual, 2 de diciembre de 2004, disponible en https://bit.ly/3oE2yVg. 
por cualquier otro medio, ${ }^{7}$ sin limitación alguna en cuanto a la ubicación geográfica, el idioma, la forma o la antigüedad de la información divulgada. Por lo tanto, ese «todo» hecho accesible al público no debe ser suficiente para permitir la reproducción de la invención que se pretende patentar; si una persona versada en la materia es capaz de reproducir la invención con la información disponible, la invención no es patentable y, en consecuencia, se considera incorporada en el estado de la técnica (Cabanellas, 2004: 722-723; Botana Agra, 2013: 120-121).

En cuanto a la accesibilidad al público, es necesario precisar qué se entiende por ella, a quiénes se les considera «público» y cómo se produce la divulgación. La accesibilidad debe ser interpretada como una posibilidad hipotética de tener acceso a la información, sin tener en consideración los motivos o intereses por los cuales una persona no pueda acceder a ella. ${ }^{8}$ Como explica Botana Agra (2013: 121-122):

La accesibilidad en este contexto significa la mera posibilidad de que el público haya entrado en contacto o tuviera la posibilidad de acceder a la invención; posibilidad que será apreciada, siempre que sea tal, al margen de la mayor o menor probabilidad de la misma. Por tanto, la mera posibilidad de acceso por parte del público es suficiente para que la invención de que se trate se considere comprendida en el estado de la técnica, al margen de que se produjera o no el efectivo acceso o contacto al público, y con independencia de que el público haya obtenido o no conocimiento sobre la misma.

Sin embargo, tenemos que considerar que, en ciertas áreas de la ciencia, la mera posibilidad de acceder al conocimiento comprendido en el arte previo implica la existencia de tecnología sin la cual sería imposible acceder a dicho conocimiento, aun cuando éste existiese (Cisneros, 2009: 34).

Con respecto al público, se entiende que estará constituido por cualquier tercero que haya podido tener acceso a la invención o a información sobre ella, sin la obligación de confidencialidad que restrinja ni su uso ni su divulgación (Botana Agra, 2013: 122).

En atención al cómo se produce la divulgación, ${ }^{9}$ puede efectuarse: 1) mediante una

7. Ello significa que no se trata sólo de información, sino que también puede incluirse la sustancia, el material o el producto que contenga la invención que, como en el caso de la nanotecnología, haya sido puesto a disposición del público previo a la solicitud de la patente (Cisneros, 2009: 31).

8. Por ejemplo, en In re Hall, 781 F.2d 897, 898 (Fed. Cir. 1986) se pone de manifiesto que en Estados Unidos lo accesible tiene un estándar bajo de cumplimiento. En el caso discutido en la referida decisión, se mantuvo que una simple copia de una tesis doctoral que formaba parte del fondo bibliotecario de una universidad alemana, y que sólo estaba disponible en ese lugar y en idioma alemán, constituía arte previo en Estados Unidos, a pesar de las dificultades de acceso.

9. Debido a que la doctrina estadounidense es extensa en este tema, y establece una de las principales diferencias con los sistemas continentales, expandiremos la presentación de la forma en que se entiende 
publicación en forma tangible, es decir, aquélla que ha sido respaldada por medio escrito; ${ }^{10} 2$ ) a través de la venta o comercialización de un producto o servicio, siempre y cuando ésta «tenga la potencialidad para hacer a la tecnología accesible al público» (Cabanellas, 2004: 705), ya sea por medio de la observación o ingeniería inversa de dicho producto o servicio; 3 ) mediante el uso de la invención por terceros no sujetos a la obligación de confidencialidad; 4) por medio del contenido de las solicitudes de patentes o modelos de utilidad presentados con anterioridad ante las oficinas de patente, aunque no hayan sido publicadas, con el objetivo de evitar que se tramiten, respecto de una misma invención, dos patentes en forma simultánea; y 5 ) «por cualquier otro medio», lo que puede entenderse, como lo hizo el juez de la Corte Suprema de los Estados Unidos, Clarence Thomas, ${ }^{11}$ que esta expresión "paraguas» no abarcaría otros medios, sino aquellos asimilables a los primeros cuatro casos.

Por último, el momento relevante como elemento de delimitación de la novedad se determina cuando se hace la solicitud de patente ante la autoridad administrativa respectiva. Esta fecha permite fijar el momento que define el estado de la técnica y el contenido de la invención que ha de contrastarse con lo incorporado al estado. No obstante, la excepción de divulgación inocua, como veremos más adelante, cambia el momento en el que se evalúa el estado de la técnica.

Como expone Ahn (2014: 98-99), en la doctrina anglosajona el estado de la técnica debe ser capaz de permitir la reproducción explícita o inherente de la invención, de tal manera que una persona con habilidades en el campo de la invención pueda replicarla sin experimentación indebida (enablement). ${ }^{12}$ Además, es necesario evaluar la llamada «anticipación inherente»; la invención se anticipa en la divulgación cuando ella es el resultado natural que emerge de la divulgación explicita en la técnica subyacente. El estado de la técnica depende también del desarrollo de la invención: es más difícil determinar que las invenciones mejoradas están en ella, dada la acumu-

la divulgación en la doctrina estadounidense en la sección «Estados Unidos», referida a la forma y supuestos con que la legislación de dicho país establece la excepción de divulgación inocua. No obstante, en lo esencial, el 35 U.S.C. 102 (a) consagra la divulgación en términos similares a los descritos en esta sección.

10. Si bien la Directrices de Examen y Procedimiento de Registro de Patentes de INAPI (p. 162) no son vinculantes, ponen de manifiesto un esfuerzo por aportar reglas con respecto a lo que se entiende por divulgación. Esta forma de definir el término, es decir, mediante el respaldo por medio escrito de lo divulgado, tiene su base en un proceso armonizador llevado a cabo en el contexto del Tratado de Cooperación en Materia de Patentes (PCT, por sus siglas en inglés) (regla $33.1 \mathrm{~b}$ ).

11. Caso Helsinn Healthcare S.A. con Teva Pharm. USA, Inc., 139 S. Ct. 628, 634 (2019).

12. La experimentación se considera indebida cuando es necesaria más experimentación de la que es establecida en la revelación de la invención. Si una persona versada en la materia relacionada con la invención no puede reproducir la invención con la información aportada, entonces no hay revelación y, por tanto, no debería otorgarse la patente. Véase In re Wands, 858 F.2d 731, 737, 8 USPQ2d 1400, 1404 (Fed. Cir. 1988). 
lación de arte previo en el tiempo. La complejidad del área de desarrollo de la invención, como ya dijimos, es otro factor que dificulta la determinación de su pertenencia al estado de la técnica.

Parte de la doctrina continental (Cabanellas, 2004: 700-701; Fernández-Nóvoa, Manuel Otero y Botana Agra, 2013: 119; Martínez, 2014: 99-101) ha señalado que la novedad se considera como: i) objetiva, puesto que se verifica a través de un proceso de comparación entre el estado de la técnica existente al momento de efectuar la solicitud de patente, y la invención reivindicada; ii) única y completa, en otras palabras, para que el estado de la técnica revele la insuficiencia de la novedad de una invención, es necesario que en él se contengan documentos que incluyan información de la cual se derive, de manera directa y sin ambigüedad, el objeto de que trata la reivindicación, por lo que no está permitida la denominada interpretación estilo «mosaico»; ${ }^{13}$ y iii) la pérdida de la novedad es irreversible, puesto que cualquier información que forme parte del estado de la técnica trae como consecuencia que la invención pierda su carácter de novedosa.

Existe un cuarto elemento relacionado con la exigencia geográfica del requisito de novedad. Las legislaciones en materia de patentes se agrupan en torno a tres definiciones de la territorialidad de la novedad (Ahn, 2014: 96-97). En primer lugar, las que consideran que la novedad debe ser absoluta o universal, es decir, que la invención no debe existir en ninguna parte del mundo. Definiciones de novedad como la encontrada en la Ley de Propiedad Industrial chilena (artículo 33 de la Ley 19.039) y la Ley de Patentes española (artículo 4 de la Ley 24/2015) encajarían en este grupo. En sistemas de patente de tipo comunitario, como el europeo, ${ }^{14} \mathrm{el}$ andino ${ }^{15} \mathrm{o}$ el internacional, ${ }^{16}$ también se define la novedad en términos absolutos o universales.

En segundo lugar, se encuentran las que consideran que la novedad debe ser local, es decir, que la invención no debe estar comprendida en el estado de la técnica del país en el que se hace la solicitud de la patente. Históricamente, lo relevante era que la invención no se conociera en el país en el cual se quería impulsar un desarrollar un área particular de la industria. Por ejemplo, en 1406, la República de Florencia le concedió a Guerinus de Mera una patente que le permitiera instalar su fábrica de cerdas para máquinas de cardar lana, a la vez que se le pidió que enseñara el funcionamiento en el territorio. Por su parte, la República de Venecia recompensó en 1416 a Franciscus Petri con una patente para desarrollar batanes de mayor rendimiento,

13. OMPI, «Ampliación...». Se refiere a una técnica de interpretación, en virtud de la cual se combinan elementos contenidos en diversas anterioridades para afectar la novedad de una invención.

14. Artículo 54, parágrafo 2 del Convenio de Múnich sobre la Patente Europea.

15. Artículo 16 de la Decisión 486 del Régimen Común sobre Propiedad Industrial de Comunidad Andina.

16. Regla 33.1 letra a) del Reglamento del Tratado de Cooperación en Materia de Patente (TCP/PCT). 
mientras que en 1469 le confirió a Johan Speyer otra para implementar una nueva técnica de impresión de libros. ${ }^{17}$ Esta práctica de otorgar privilegios para desarrollar la industria local luego se fue extendiendo hacia otros países del continente europeo (Fernández López, 2009: 254), e incluso se mantuvo en algunos países en el siglo XX bajo la etiqueta de «patentes de introducción». ${ }^{18}$

En tercer lugar, se hallan aquellas legislaciones que consideran que la novedad es mixta, es decir, que la publicación escrita en cualquier parte del mundo anula la novedad, pero localmente la anula la publicación oral o el uso de la invención. Éste es, como veremos, el caso del sistema de patentes de estadounidense. ${ }^{19}$

\section{La divulgación inocua de la invención como excepción al requisito de novedad en el derecho comparado}

Algunos países han incorporado en sus legislaciones de patente instituciones jurídicas que constituyen una excepción a la exigencia de novedad de la invención, estableciendo que, si la divulgación de la invención se produce en determinados supuestos, no pasará a formar parte del estado de la técnica y, en consecuencia, la invención no perderá su carácter novedoso.

La novedad de la invención puede ser inmune a: i) las divulgaciones hechas en exposiciones nacionales o internacionales oficiales $\mathrm{u}$ oficialmente reconocidas, ${ }^{20} \mathrm{ii}$ ) las divulgaciones hechas con motivo o derivadas de abusos y prácticas desleales; iii) las divulgaciones autorizadas o derivadas de inventor. ${ }^{21}$ Como podemos observar, los supuestos bajo los cuales procedería la declaración de excepción de divulgación inocua son variados y el "periodo de gracia»o "protección temporal» - concebido como el espacio de tiempo durante el cual la invención divulgada es objeto de protección- también lo es.

17. Una rigurosa revisión sobre la historia de la patente puede verse en Prager (1944, 1946) y Hulme (1896). 18. Véase, por ejemplo, el artículo 12 de la Ley del 16 de mayo de 1902 de Propiedad Industrial española. 19. 35 U.S.C. $\$ 102$ (a) 1.

20. El artículo 11 del Convenio de París de 1883, que recoge la protección temporal para las invenciones expuestas en exposiciones internacionales oficiales u oficialmente reconocidas, es considerada - con sus críticas por la falta de precisión- como la disposición más original, aunque directamente vinculada con la Gran Exposición de Londres en 1851, la primera exposición internacional en su género (Ricketson, 2015: 416-420). No obstante, fue la negativa de los inventores estadounidense a participar en la Feria Mundial de Viena de 1873 - debido a la insuficiencia de la legislación austríaca para proteger sus invenciones- la que marcó el camino hacia este Convenio (Ricketson, 2015: 32-37).

21. Para más información sobre la configuración de los supuestos de inocuidad de la novedad en el orden internacional, se recomienda ver el reporte «Informe sobre el Sistema Internacional de Patentes: Anexo II revisado del documento», SCP/18/2 (anexo sobre periodo de gracia), elaborado por el Grupo de Trabajo en materia de patentes de la Organización Mundial de la Propiedad Industrial disponible en https://bit.ly/31NmN8Y. 
No hay un estándar internacional con respecto al límite de la protección temporal: más de cincuenta países en el mundo contemplan en su legislación la figura del periodo de gracia de la invención patentable. Por ejemplo, en Argentina, Brasil, Canadá y Singapur, el periodo de protección es de doce meses; en Alemania, Austria, Bélgica, Bulgaria, Portugal, Suecia y Suiza, dicho periodo es de seis meses; en Australia es de seis o doce meses dependiendo de los supuestos bajos los cuales procede la divulgación inocua, mientras que en Sudáfrica se recurre a la «fecha de prioridad», por lo que será de doce meses. ${ }^{22}$

Según Merges (2012), el fundamento que subyace en el periodo de gracia es la protección temporal que se le ofrece a quien inventa contra sus competidores, cuando está cercano a introducir una solicitud de patente. No obstante, Franzoni y Scellato (2010: 204) cuestionan esta institución, pues quien desee hacer una divulgación temprana de la invención y hacer uso del referido periodo de gracia deberá tener un amplio conocimiento de su configuración en el ámbito nacional e internacional antes de divulgar, sobre todo si lo que pretende es una protección en más de un país. En una interpretación a contracorriente, Joachim (2015: 1.298) considera que el periodo de gracia se establece como una herramienta que facilita verificar la garantía de la novedad de una invención, pues - como ya hemos expuesto- el requisito de novedad puede resultar impracticable a nivel absoluto o universal en ciertas áreas de la ciencia y la tecnología ${ }^{23}$ al publicar antes de introducir la solicitud de una patente, se permite al público conocer la invención y determinar de manera más adecuada si es o no nueva. Por tanto, el escepticismo organizado, ese imperativo categórico mertoniano que contribuye en la «certificación» formal de la ciencia, podría hacer de la divulgación temprana de la invención la principal colaboradora en la verificación de la novedad para el sistema de patentes.

\section{Chile}

La Ley de Propiedad Industrial chilena contempla, al igual que muchas legislaciones a nivel mundial, diferentes supuestos bajo los cuales la divulgación de la invención no afecta el requerimiento de novedad. El artículo 42 de la Ley 19.039 establece dos supuestos. El primero está relacionado con las divulgaciones hechas o derivadas de prácticas desleales que hubieren afectado al solicitado: en el transcurso del desarrollo de una investigación que da lugar a resultados potencialmente patentables intervienen múltiples actores que podrían divulgar la invención sin consentimiento del inventor o propietario. El segundo está relacionado con la divulgación hecha, autorizada o derivada del solicitante de la patente. En ambos supuestos, la Ley de Propiedad

22. OMPI, «Informe...», anexo.

23. El caso más paradigmático es el ya mencionado In re Hall, 781 F.2d 897, 898 (Fed. Cir. 1986). 
Industrial chilena protege al inventor, manteniendo su derecho a solicitar la patente, siempre que haga la solicitud durante el periodo de gracia.

La obligación de contemplar la protección temporal de la invención para el caso de las exposiciones internacionales no se reconoció en Chile sino con la suscripción del Convenio de París, en 1991. En 2005 se materializó esta obligación, junto a otros dos supuestos, cuando se modificó el artículo 42, mediante la reforma introducida por la Ley 19.996 a la Ley 19.039 de 1991, para incluir la divulgación inocua en la legislación interna. Esta modificación contemplaba una periodo de gracia de seis meses para divulgaciones que fueran consecuencia directa o indirecta de: a) las prácticas, ensayos y construcción de mecanismos o aparatos que deba hacer el solicitante que tenga una invención en estudio; b) las exhibiciones del invento hechas por el solicitante o su causante en exposiciones oficiales u oficialmente reconocidas; o c) los abusos y las prácticas desleales de las que hubiere sido objeto el solicitante o su causante.

La Ley 20.160 de 2007 incluyó nuevas modificaciones a la Ley 19.039 de Propiedad Industrial para cumplir con los compromisos adquiridos en el orden internacional por Chile. ${ }^{24} \mathrm{El}$ artículo 42 fue modificado para ampliar el periodo de gracia a doce meses anteriores a la fecha de presentación de la solicitud de patente, y se redujo a dos los supuestos bajo los cuales las divulgaciones tempranas pueden considerarse inocuas. Se suprimen así los supuestos considerados en las letras a) y b), incorporando en su lugar el siguiente texto: «Si la divulgación pública: a) fue hecha, autorizada o deriva del solicitante de la patente». De esta forma, la ley chilena amplía el espectro de situaciones y evita establecer categorías taxativas de éstas para ir más allá de las derivadas de ensayos, prácticas o exposiciones en eventos internacionales, como lo hacía antes, lo que incluye las publicaciones científicas.

Sobre el supuesto que se contemplaba en el literal c) del artículo 42 de la Ley 19.039 de Propiedad Industrial, la modificación se establece casi en los mismos términos en la nueva letra b) incorporada en la Ley 20.160. ${ }^{25}$ Este régimen se encuentra vigente hasta el día de hoy, dado que la modificación a la Ley 19.039 de Propiedad Industrial del año 2012, introducida por la Ley 20.569, en nada modificó la referida institución, aunque es importante destacar que tampoco se contemplan modificaciones a ella en el actual proyecto de reforma de la Ley 19.039 de Propiedad Industrial que se discute en el Congreso. ${ }^{26}$

En cuanto a la oportunidad y procedimiento para reclamar y acreditar la existen-

24. Historia de la Ley 20.160 que modifica la Ley 19.039, de Propiedad Industrial.

25. Con esta modificación se da cumplimento a lo acordado en el artículo 17.9 número 7 del tratado de libre comercio suscrito entre Chile y Estados Unidos en junio de 2003, y que entró en vigor el 1 de enero de 2004. Véase también la Ley 20.160, de 2007.

26. Proyecto de ley 20.254 del 20 de septiembre de 2018, que modifica la Ley 19039 de Propiedad Industrial. Disponible en https://bit.ly/3oAkPm1. 
cia de una divulgación inocua, el reglamento de la Ley 19.039, en sus artículos 37 y 58 inciso segundo, señala que, cuando se alegue la existencia de las divulgaciones mencionadas en el artículo 42 de la Ley, deberá acompañarse la solicitud de patente con un escrito que permita verificar los elementos objetivos de la divulgación realizada. ${ }^{27}$

\section{España}

España, al igual que Chile y Estados Unidos, con la suscripción del Convenio de París en 1883 se comprometió a conceder una protección temporal de seis meses a las invenciones patentables que se exhibiesen en exposiciones internacionales oficiales $\mathrm{u}$ oficialmente reconocidas. ${ }^{28}$ No obstante, la regulación, hablando propiamente en materia de propiedad industrial, sólo se alcanzó con la Ley del 16 de mayo de 1902 (Rodríguez, 2016: 12). En la referida ley se amplía a dos los supuestos de protección temporal de seis meses a las invenciones patentables, al incluir los ensayos previos a la solicitud de la patente, siempre que la divulgación hubiese provenido del inventor o sus derechohabientes. ${ }^{29}$

Luego, en la Ley 11/1986 de Patentes se incluyó, además, que los ensayos hechos tanto por el solicitante como sus causahabientes no deberían implicar una explotación comercial $u$ ofrecimiento comercial del invento (artículo 7 letra c). Por otra parte, se incorporó, en armonía con el artículo 55 letra a) del Convenio sobre la Concesión de Patentes Europeas de 1973, un tercer supuesto de protección temporal de seis meses para las divulgaciones de la invención derivadas de prácticas abusivas en contra del inventor o de su predecesor legal..$^{30}$

27. No obstante, la Corte Suprema de Chile consideró que «la inobservancia de este presupuesto simplemente reglamentario [el acompañamiento de la solicitud de patente de los documentos que acrediten la existencia, naturaleza y fecha de las divulgaciones] no puede producir un efecto tal que impida examinar el negocio de fondo anunciado en la solicitud. Desde luego, porque esa abstención carece de una sanción concreta asignada en el cuerpo reglamentario, y en seguida, porque con independencia de la fórmula utilizada, el peticionario esgrimió el talón D8 como una divulgación inocua y antecedente de su pretensión, de suerte que aparece inconducente la omisión de resolver el punto por una mera cuestión formal y, de contrario, se hace procedente un pronunciamiento sobre el fondo». Causa 26853/2014 (otros), resolución 88.267 de la Corte Suprema, Sala Segunda (Penal) del 10 de junio de 2015.

28. Real Decreto del 19 de agosto de 1888, Gaceta de Madrid núm. 32. Disponible en https://bit. ly/30DRxmz. Para un análisis más detallado sobre la historia de la Ley de Propiedad Industrial española, se recomienda ver la compilación de Sáiz (1996).

29. Artículo 15 de la Ley del 16 de mayo de 1902, que se mantuvo en el artículo 59 del Estatuto sobre Propiedad Industrial de 1929, que vino a reformar la Ley de 1902 y su reglamento del 15 de enero de 1924.

30. Botana Agra (2013) ha sostenido que la exigencia de que el abuso sea evidente ante el solicitante o su causahabiente, implica que la protección temporal está reservada para los casos de abuso más extremos, por ejemplo, para aquéllos derivados del incumplimiento de obligaciones de mantener en secreto la información revelada. 
Sin embargo, el referido supuesto relacionado con los ensayos hechos por el inventor o su causahabiente que no impliquen la explotación comercial del invento fue suprimido mediante la Ley 24/2015 de Patentes que entró en vigor en España el 1 de abril de 2017. La supresión de la referida excepción de divulgación inocua se ha basado en la inexistencia de ésta en el derecho comparado europeo y, en consecuencia, mantenerla podría afectar la novedad de la invención si se quiere ampliar su protección a países que no reconocen en su legislación dicha protección temporal. ${ }^{31} \mathrm{Algu}-$ nos autores han sostenido que la excepción a la novedad relacionada con los ensayos (artículo 7 de la Ley 11/198) era «una incomprensible e injustificada singularidad del derecho español en materia de divulgaciones inocuas» (Gómez Segade, 2014: 342). Por contra, hay quienes sostienen que la eliminación del supuesto «entorpece la labor de transferencia exigida a las universidades y, en todo caso, hace que los investigadores deban ser todavía más cautelosos con sus resultados» (Sánchez García, 2017: 8).

Por último, en cuanto al procedimiento para reclamar una divulgación como inocua, el reglamento sancionado para la implementación de la Ley 24/2015 de Patentes sólo se refiere a las divulgaciones derivadas del artículo 7.b de la Ley, y señala en su artículo 2.2.f que en la instancia de solicitud de patente deberá indicarse el nombre, lugar y fecha en que tuvo lugar la exhibición de la que deriva la divulgación.

Por tanto, en España no se reconoce la excepción de divulgación inocua en los casos en que los investigadores universitarios deseen hacer una comunicación científica de los resultados de una investigación, antes de la solicitud de una patente referida a dichos resultados.

\section{Estados Unidos}

Para no romper con el discurso inicial, centrado en la configuración de la novedad bajo el sistema jurídico de influencia continental, hemos querido dejar aparte la definición de novedad en la legislación estadounidense. Ya en 1793 se establecía estatutoriamente la necesidad de la novedad en la solicitud de una patente. No obstante, lo que constituía una invención nueva no era definido, por lo que, a lo largo de la historia jurisprudencial estadounidense, y a partir de la Patent Act de 1836, se estableció como criterio para determinar la novedad el denominado «punto de novedad» (point of novelty). ${ }^{32}$ Éste es el teórico punto en el que se podía diferenciar lo que hacía nueva la invención de lo que ya existía en el estado del arte, a pesar de que dicha invención pudiese usar elementos de éste en su diseño (Burk y Lemley, 2009a). Esta doctrina consideraba, primero, si las reivindicaciones de una solicitud de patente no eran una

31. Véase el preámbulo de la Ley 24/2015 de Patentes, parte 2.

32. En ella se establecía que el inventor debía especificar y apuntar en particular la parte, mejora o combinación que la reivindicaba como invención o descubrimiento propio. Patent Act de 1836, $₫ 6$. 
mezcla conocida de elementos del estado del arte que cualquiera con habilidad en la materia pudiese haber hecho (law of obviousness), y segundo, si ninguna referencia del estado del arte contenía todas las reivindicaciones de dicha solicitud (anticipation). Las patentes se redactaban, entonces, con reivindicaciones Jepson, aquellas en las que se establecía el punto de novedad, lo que aseguraba que no se confundiera el estado del arte con lo que auténticamente se quería patentar. ${ }^{33} \mathrm{El}$ problema, como expone Lemley (2011), es que casi todas las invenciones serían de tipo Jepson, por lo que muy pocas patentes se otorgarían sobre invenciones completamente nuevas y la gran mayoría serían mejoras de invenciones existentes. Por esa razón, las cortes del circuito federal estadounidense fueron dejando de lado el punto de novedad como criterio, prefiriendo la revisión de la invención como un todo. ${ }^{34}$ El lenguaje de redacción de las reivindicaciones pasó entonces a tomar un rol preponderante, proceso que se aceleró a partir de 1996, cuando la Corte Suprema de los Estados Unidos decidió que constituía una cuestión sobre la que los tribunales federales deberían decidir, ${ }^{35}$ lo que hizo que las reivindicaciones Jepson bajasen del $15 \%$ del total en 1980 a menos de $1 \%$ en 2008 (Lemley, 2011).

En la actualidad, la novedad se configura estatutoriamente en la sección 102 del Código de los Estados Unidos (35 U. S. C. \$ 102). Mediante la subsección 35 U. S. C. $\$ 102$ (b), la Patent Act de 1952 estableció un límite estatutario (statutory bar), que puede entenderse como una definición implícita de período de gracia. ${ }^{36}$ No obstante, este periodo era definido en forma negativa, pues determinaba el momento a partir del cual se perdía el derecho a solicitar la patente. Dicho periodo era de doce meses para la divulgación de las invenciones que fueron patentadas o descritas en una publicación impresa dentro o fuera del país, o que fueran objeto de uso público o venta dentro del mismo país.

Esta manera de regular la divulgación inocua estuvo vigente en Estados Unidos hasta el año 2011, cuando se sancionó la America Invents Act, que modifica la sección

33. Ex parte Jepson, 1917 Dec. Comm'r Pat. 62, 67-68.

34. Rosemount, Inc. con Beckman Instruments, Inc., 727 F.2d 1540, 1543 n.3 (Fed. Cir. 1984); Loctite Corp. con Ultraseal Ltd., 781 F.2d 861, 875 (Fed. Cir. 1985); Bausch \& Lomb, Inc. con BarnesHind/Hydrocurve, Inc., 796 F.2d 443, 449 (Fed. Cir. 1986); Para-Ordnance Mfg., Inc. con SGS Imps. Int'l, Inc., 73 F.3d 1085, 1087 (Fed. Cir. 1995). En el caso de las patentes de diseño, la corte de Egyptian Goddess, Inc. con Swisa, Inc. sustituyó este examen por el de «observador ordinario» (ordinary-observer), en el que la corte se pregunta si éste sería capaz de decidir que los dos diseños en juicio fuesen el mismo. Véase Egyptian Goddess, Inc. con Swisa, Inc., 543 F.3d 665, 677 (Fed. Cir. 2008).

35. Markman con Westview Instruments, Inc., 517 U.S. 370, 372 (1996); Cybor Corp. con FAS Techs., Inc., 138 F.3d 1448, 1451 (Fed. Cir. 1998).

36. Aunque en la Patent Act de 1839 se establecía ya un periodo de gracia para publicaciones o usos de la invención de 24 meses $(\$ 10)$. 
102 para reconocer, de forma expresa y mucho más clara, esta institución, ${ }^{37}$ adoptando los supuestos que consagran el modelo oficialmente denominado como firstinventor-to-file. Según el actual texto de la Ley, en su subsección \$102 (b)(1)(A), el periodo de gracia en los Estados Unidos es de doce meses, y eliminará del estado de la técnica una divulgación pública bajo el supuesto de que ésta sea hecha por el propio inventor o por terceros, cuando éstos han obtenido el objeto de protección (subject matter) de manera directa o indirecta del inventor. Se entiende que la divulgación del objeto de protección hecha por terceros sin autorización del inventor no formará parte del estado del arte y activará el periodo de gracia, según lo establecido en la $\$ 102(b)(1)(A)$. Para garantizar que cualquier divulgación active el periodo de gracia, el inventor deberá incluir una declaración de inocuidad según lo que establece la U.S. Patent \& Trademark Office (USPTO) (2014) en las subsecciones \$2153.01(a) y (b) de su manual de examinación de patentes. De lo contrario, se considerará la divulgación como parte del estado del arte y, por lo tanto, el inventor no podrá argumentar la novedad de la invención. Por otra parte, y de acuerdo a Merges, Menell y Lemley (2012: 255-257), el periodo de gracia de la subsección \$102 (b)(1)(B) configura un supuesto más cercano a la novedad absoluta o universal y a la ausencia de periodo de gracia establecida por otros sistemas de patentes, como el europeo, pues la divulgación del objeto de protección por terceros hecha antes de aquélla hecha por el inventor anula la novedad. En el sentido de la divulgación inocua como incentivo a la divulgación temprana de la invención, la configuración de los supuestos de la \$102 parecen dar vida a un sistema que de manera más adecuada debería ser denominado como firstinventor-to-disclose, por la importancia que se le da a la divulgación inicial como fecha de prioridad en el proceso de solicitud de una patente.

La principal diferencia entre otras formas de regulación de la institución de la divulgación inocua y la forma establecida en los Estados Unidos consiste en lo que ha de entenderse por divulgación, pues para Estados Unidos la comprensión del término es mucho más amplia. En la legislación estadounidense se ha sostenido que al mantener el término divulgación utilizado de la Ley de 1952 en la redacción de la America Invents Act, se quiso incorporar una larga historia jurisprudencial relacionada con la interpretación de dicho término (Menell, 2013: 77; Merges, 2012: 1.033). En este sentido, la divulgación debe entenderse como «cualquier divulgación», aun cuando

37. 35 U.S.C. $\$ 102$ (b): «Exceptions: (1) disclosures made 1 year or less before the effective filing date of the claimed invention. $-A$ disclosure made 1 year or less before the effective filing date of a claimed invention shall not be prior art to the claimed invention under subsection (a)(1) if- (A) the disclosure was made by the inventor or joint inventor or by another who obtained the subject matter disclosed directly or indirectly from the inventor or a joint inventor; or (B) the subject matter disclosed had, before such disclosure, been publicly disclosed by the inventor or a joint inventor or another who obtained the subject matter disclosed directly or indirectly from the inventor or a joint inventor». 
esta se haga en el ámbito privado, como las «ventas confidenciales», pues se alejan del secreto ${ }^{38}$ En cambio, en otras legislaciones, como la chilena, el término divulgación está relacionado con la idea de acceso público de la información, excluyendo de él las revelaciones que se hagan en el marco de conversaciones o negociaciones privadas y confidenciales.

Otro tipo de divulgación es la llamada «divulgación no informativa», es decir, aquélla hecha mediante el funcionamiento de la invención que no es capaz de informar al público sobre su naturaleza. De acuerdo con la tradición jurisprudencial estadounidense, la divulgación no informativa hecha por el inventor se considerará arte previo y entrará en los supuestos de la $\$ 102$ (b)(1)(A), activando el periodo de gracia (Merges, 2012: 1.035).

Finalmente, un punto relevante en la jurisprudencia estadounidense sobre el tema de la divulgación es la hecha por terceros con posterioridad a la hecha por el inventor (intervening disclosures). Aunque los tribunales estadounidenses no suelen dar una deferencia Chevron ${ }^{39}$ a la USPTO, ésta anunció en $2012^{40}$ su interpretación de la $\$ 102(b)(1)(B)$, para explicar que el objeto de protección al que se hace referencia en los casos de divulgación posterior por terceros debe ser el mismo que el divulgado antes por el inventor para garantizar los derechos del inventor durante el periodo de gracia. Sin embargo, meros cambios insustanciales, o sólo variaciones triviales o insustanciales del objeto de protección, no activan la excepción, sino que las incorporan directamente en el arte previo. Esta interpretación llevó, como Joachim (2015) describe, a la protesta generalizada de diferentes actores relacionados con la patente, incluyendo cientos de universidades estadounidenses, y a cambios en la interpretación hechos por la USPTO en 2013 para aclarar que no hay ningún requisito de que la divulgación por parte del inventor o coinventor deba ser literal a la posterior por terceros, a fin de que la excepción se active. ${ }^{41}$

38. Estas ventas configuran el denominado «límite sobre las ventas» (on-sale bar), una prohibición explicita de patentar la invención si ha estado en venta por más de un año. Ésta fue originalmente establecida de manera jurisprudencial antes que estatutoria en Pennock con Dialogue, 27 U.S. 1, 2 Pet. 1 (1829). Incluso, el juez Thomas, en la redacción del fallo unánime de la Corte Suprema de Estados Unidos del caso Helsinn Healthcare S.A. con Teva Pharm. USA, Inc., 139 S. Ct. 628, 634 (2019), concluyó que el Congreso de los Estados Unidos no tuvo la intención de modificar la configuración legal del «límite sobre las ventas» al dictar la America Invents Act, es decir, cualquier venta activa el periodo de gracia. Para un análisis completo sobre la decisión, véase Howes (2020).

39. Una deferencia Chevron se refiere a un principio de derecho administrativo por el que los tribunales estadounidenses defieren la interpretación de normas ambiguas a las agencias federales que las administran. Véase Chevron U.S.A., Inc. con Natural Resources Defense Council, Inc., 467 U.S. 837 (1984). 40. «Examination guidelines for implementing the first-inventor-to-file provisions of the LeahySmith America Invents Act», 77 Fed. Reg. 43,759 (26 de julio de 2012).

41. «Changes to implement the first inventor to file provisions of the Leahy-Smith America Invents Act», 78 Fed. Reg. 11,024 (14 de febrero de 2013); «Examination guidelines for implementing the first 


\section{Discusión y conclusiones}

Entendiendo que Chile, España y Estados Unidos suscribieron el Convenio de París como el primer intento internacional de armonización del derecho de la propiedad industrial, es importante destacar que la legislación interna de dichos países tiene marcadas diferencias en la forma de regularla. La excepción de divulgación inocua es un ejemplo de dichas discrepancias que dan cuenta de la complejidad del proceso armonizador.

En cuanto a los supuestos bajo los cuales procedería la protección temporal de la novedad de la invención, se puede observar que al menos la divulgación de invenciones hecha en exposiciones internacionales es un estándar seguido por los países estudiados y, tal vez, ello sea una consecuencia del contexto histórico en el que surge. ${ }^{42}$ Los otros dos supuestos - relacionados con las divulgaciones con motivo o derivadas de abusos y prácticas desleales, y las divulgaciones autorizadas o derivadas del inventor- han seguido un camino más tortuoso en su armonización. Aunque estos supuestos pudieran llegar a armonizarse de manera positiva -como ocurre con el establecido en el artículo 11 del Convenio de París-, la excepción de divulgación inocua sería inútil en la práctica si el periodo de gracia o de protección de la novedad de la invención se establece de forma diferenciada o aislada en cada país. Supongamos que un investigador quiere cumplir con el imperativo categórico mertoniano del comunitarismo comunicando los resultados de su investigación tan pronto como los obtenga. Si, además, quiere patentar dichos resultados, deberá asegurarse de que las legislaciones en materia de patentes de los países en los que busca protección contemplan un periodo de gracia en los supuestos relacionados a la divulgación científica.

Asimismo, deberá identificar claramente cuál es la duración de dichos periodos de gracia, lo que le llevará a tener que considerar que su periodo de gracia es, a todos los efectos, el menor de todos. Para las legislaciones chilena y estadounidense, cualquier divulgación hecha o autorizada por el inventor durante los doce meses anteriores a la fecha de solicitud de patente quedará excluida del estado de la técnica, sin limitar el contexto en el que ellas se hiciesen. Por el contrario, hasta abril del 2017, la legislación española contemplaba la excepción para las divulgaciones que se derivaran de ensayos hechos por el solicitante; sin embargo, esta excepción ya no se contempla de esta forma en la actual normativa vigente. ${ }^{43}$ Debido a que la legislación española

inventor to file provisions of the Leahy-Smith America Invents Act», 78 Fed. Reg. 11,059 (14 de febrero de 2013). En este sentido, un trabajo previo a la America Invents Act por Franzoni y Scellato (2010) muestra cómo los resúmenes de pares de publicaciones-patentes pueden tener diferentes grados de similitud, medidos objetivamente con algoritmos, lo que soportaría la nueva interpretación de la USPTO.

42. Véase nota 20.

43. Ley 24/2015, del 24 de julio, de Patentes (Boletín Oficial del Estado núm. 177, del 25 de julio de 2015). 
dejó de ser una singularidad en el entorno europeo con respecto a este tema, surge la duda de si un sistema sin periodo de gracia es más beneficioso para los investigadores universitarios que uno que sí lo contemple. De hecho, un reporte de la ScienceBusiness Innovation Board (Edmonson y otros, 2013) revela que los administradores de oficinas de transferencia tecnológica consideran que los investigadores europeos se encuentran en desventaja vis- $a$-vis con sus contrapartes estadounidenses - o chilenas, por ejemplo-, ya que cualquier revelación anula la novedad. Esta desventaja, según los entrevistados para este reporte, desalienta la innovación, pues la publicación de resultados de investigación es prioritaria en la carrera académica, además de que fuerza a las universidades a presentar solicitudes que posiblemente no estén tan refinadas como si el periodo de gracia se los permitiese lograr.

Discrepamos con esta afirmación, pues a pesar del periodo de gracia, un investigador debe determinar, ex ante, si sus resultados podrían ser patentados; si decide publicar, debe recordar que el periodo de gracia se activa con la publicación. Reconocer la patentabilidad de un resultado de investigación puede no ser una tarea fácil u obvia, por lo que, si se logra después de la publicación, tal vez el periodo de protección otorgado sea poco para conseguir una forma completa de invención patentable y cumplir con el procedimiento necesario para solicitar la patente. Por tanto, habría poco incentivo a invertir la lógica tradicional «patentar y luego publicar». En el caso de Chile, hemos podido identificar al menos cuatro solicitudes de patente $\mathrm{e}^{44}$ en que se ha puesto de manifiesto que, en efecto, el periodo de gracia es considerado en la evaluación de las solicitudes de patentes hechas por universidades. La primera fue denegada por existir una publicación del equipo de investigación que no fue reportada como divulgación inocua, la segunda por haberla hecho fuera del plazo legal (doce meses), mientras que en las dos últimas se hizo la declaración de manera adecuada. ${ }^{45}$

Antes de la aparición de la America Invents Act, Franzoni y Scellato (2010: 206) encontraron que sólo el $27,4 \%$ de las solicitudes de patentes con origen académico

44. Solicitud de patente de invención 2012-000765, del 28 de marzo de 2012, de la Universidad de La Frontera; solicitud 2014-02394, del 10 de septiembre de 2014, de la Universidad de Chile; solicitud 201301531, del 30 de mayo de 2013, de la Universidad de Tarapacá; solicitud 2016-03233, del 16 de diciembre de 2016, de la Universidad de Concepción.

45. Gracias al personal del INAPI fue posible obtener esta pequeña muestra de casos relevantes para este trabajo. No obstante, las búsquedas deben ser hechas de manera manual o mediante el número del expediente, por lo que un trabajo extenso requerirá mucho tiempo. En el caso estadounidense, a través de Google Patents (http://patents.google.com) es posible identificar el uso del periodo de gracia de los documentos de patente digitalizados por esta empresa. Sin embargo, el formato de declaración del arte previo divulgado por el inventor no está estandarizado y complica la búsqueda a los fines de investigación. La falta de estandarización está contemplada por la U.S. Patent \& Trademark Office en la subsección \$2153.01(a) del «Manual of patent examining procedure» (disponible en https://bit.ly/3e8QRRm), su manual de examinación de patentes, que no la requiere para hacer su evaluación de novedad. 
presentadas ante la USPTO United States Patent and Trademark Office hacían uso del periodo de gracia. Por su parte, el estudio realizado por el Grupo Tegernsee ${ }^{46}$ evidencia que el $67 \%$ de los investigadores estadounidenses entrevistado y el $64 \%$ de los europeos sentían la necesidad de presentar una solicitud de patente de forma posterior a una divulgación, principalmente en el contexto de eventos académicos. No obstante, los problemas relacionados con la pérdida de la novedad que hemos señalado inducen a recurrir a esta figura jurídica en una proporción muy baja con respecto del total. El estudio del Science-Business Innovation Board (Edmonson y otros, 2013) concuerda con los resultados del Grupo Tegernsee, pues $67 \%$ de los investigadores europeos entrevistados apoyan la implementación de un periodo de gracia de doce meses en la Unión Europea para nivelar la situación con respecto a sus pares norteamericanos. El efecto de la falta de período de gracia en la Unión Europea puede observase en sus aplicaciones de patentes en Estados Unidos, las que se hacen sólo como una medida de último recurso cuando existe una publicación que anula la novedad. Por último, entre los entrevistados de este estudio existe una visión equitativa de las ventajas del periodo de gracia, entre aquéllos que le otorgan valor por su contribución al ejercicio de la libertad académica y aquéllos que entienden que se podría evitar la pérdida de beneficios económicos y sociales de la invención. En el ámbito académico, preferimos la primera razón a la segunda, pues no deberían crearse figuras que incentivaran a la ambivalencia en el quehacer científico.

En resumen, no existe una fórmula idónea de concreción de la excepción de divulgación inocua, dados los problemas inherentes entre el sistema de patente y la ciencia académica. Sin embargo, hay que considerar que la hibridación que permite el intercambio académico es el auténtico motor del desarrollo científico. Aunque el periodo de gracia no sea la bala de plata para los problemas que la patente induce en el mundo académico (Wong, 2015), concordamos con Margo Bagley (2008) en que la inclusión de esta figura jurídica en diferentes sistemas legislativos puede verse como una mejora incremental en el proceso de armonización a nivel mundial. No obstante, el uso que los investigadores hagan de la excepción de divulgación inocua, como herramienta de equilibrio en una tensión indudable, dependerá en gran medida de que tan consecuentes sean con el ethos mertoniano, aunque también del momento en que se identifica el potencial de patentabilidad de los resultados de investigación alcanzados.

46. El Grupo Tegernsee está compuesto por las oficinas de patentes de Dinamarca, Francia, Alemania, Japón, el Reino Unido, Estados Unidos y la Unión Europea. El estudio fue preparado en el marco del inicio de los diálogos sobre los asuntos relacionados con la armonización internacional del derecho sustantivo de patente. Entre los temas claves identificados relacionados con la armonización de las leyes de patentes se incluyó el tema del periodo de gracia. "Consolidated report on the Tegernsee user consultation on substantive patent law harmonization», Tegernsee Group, mayo de 2014, disponible en https://bit.ly/2G8rJov. 


\section{Agradecimientos}

El presente trabajo fue escrito en el marco del proyecto de investigación Fondecyt de Iniciación 11150162: «Patentes e investigación Científica: Un estudio empírico sobre el ejercicio del derecho de libertad de investigación científica en las universidades chilenas que desarrollan I+D», del que la doctora Sulan Wong Ramírez fue la investigadora responsable.

Expresamos nuestro agradecimiento al personal asesor en materia de patentes del Instituto Nacional de Propiedad Industrial de Chile por la información aportada en la elaboración de este trabajo.

Damos las gracias a los revisores anónimos de este artículo por sus comentarios y sugerencias para su mejora.

\section{Referencias}

AHN, Hyewon (2014). Second generation patents in pharmaceutical innovation. Baden-Baden: Nomos Verlagsgesellschaft MbH.

BAGLEY, Margo (2006). «Academic discourse and proprietary rights: Putting patents in their proper place». Boston College Law Review, 47: 217-274. Disponible en https://bit.ly/34Cn6VR.

-. (2008). «The need for speed (and grace): Issues in a first-inventor-to-file world». Berkeley Technology Law Journal, 23: 1.035-1.061. Disponible en https://bit. ly/3kDW2v2.

Boldrin, Michele y David Levine (2008). Against intellectual monopoly. Cambridge: Cambridge University Press.

Botana Agra, Manuel (2013). «Invenciones patentables». En Carlos FernándezNóvoa, José Manuel Otero Lastres y Manuel Botana Agra (editores), Manual de la propiedad industrial. $2^{\mathrm{a}}$ ed. Madrid: Marcial Pons.

Boyle, James (2007). «Mertonianism unbound?: Imagining free, decentralized access to most cultural and scientific material». En Charlotte Hess y Elinor Ostrom (editoras), Understanding knowledge as a commons: From theory to practice. Cambridge: MIT Press.

BURK, Dan y Mark Lemley (2009a). «Fence posts or sign posts-rethinking patent claim construction». University of Pennsylvania Law Review, 157 (6): 1.743-1.799. Disponible en https://bit.ly/3jEgOto.

-. (2009b). The patent crisis and how the courts can solve it. Chicago: University of Chicago Press.

Cabanellas, Guillermo (2004). Derecho de las patentes de invención. 2. ${ }^{a}$ ed. Volumen 1. Buenos Aires: Heliasta. 
Cisneros, Mario (2009). Patentability requirements for nanotechnological inventions: An approach from the European Patent Convention perspective. Baden-Baden: Nomos Verlagsgesellschaft $\mathrm{MbH}$.

Drahos, Peter (2010). The global governance of knowledge. Nueva York: Cambridge University Press.

Edmonson, Gail, Jeremy Holmes, Rouget F. Henschel y Shane McCollam (2013). A grace period for patents: Could it help European universities innovate?. Bruselas: Science-Business Innovation Board.

FernáNDEZ López, Juan Manuel (2009). «Precedentes históricos de la protección jurídica». En María Baylos Morales (coordinadora), Tratado de derecho industrial. 3. ${ }^{a}$ ed. Pamplona: Civitas, Thomson Reuters.

FernándeZ-NóvoA, Carlos, José Manuel Otero y Manuel Botana Agra (2013). Manual de la propiedad industrial. $2^{\mathrm{a}}$ ed. Madrid: Marcial Pons.

Franzoni, Chiara y Giuseppe Scellato (2010). «The grace period in international patent law and its effect on the timing of disclosure». Research Policy, 39 (2): 200-213. DOI: 10.1016/j.respol.2009.11.006.

Gómez Segade, José (2014). «Hacia una nueva Ley de Patentes española». Actas de Derecho Industrial y Derecho de Autor, 34: 337-356.

Howes, Christopher (2020). «Helsinn Healthcare S.A. v. Teva Pharm. USA, Inc.: 35 U.S.C. $\$ 102$ looks like a new duck and sounds like a new duck, but the on-sale bar is the same ole goose». Journal of Business Technology Law Proxy, 15 (1). Disponible en https://bit.ly/3mzFCoo.

Hulme, E. Wyndha (1896). «History of the patent system under the prerogative and at common law». The Quarterly Review, 12: 141-154.

JoACHIM, Jordan (2015). «Is the AIA the end of grace?: Examining the effect of the America Invents Act on the patent grace period». New York University Law Review, 90 (4): 1.293-1.330. Disponible en https://bit.ly/3mzHkGc.

Lemley, Mark A. (2011). «Point of novelty». Northwestern University Law Review, 105 (3): 1.253-1.28o. Disponible en https://bit.ly/37SLZi4.

Machlup, Fritz y Edith Penrose (1950). "The patent controversy in the nineteenth century». The Journal of Economic History, 10 (1): 1-29. DOI: 10.1017/ So022050700055893.

Martínez, Mireia (2014). La patente biotecnológica y la OMC. Madrid: Marcial Pons. Menell, Peter (2013). «The mixed heritage of Federal Intellectual Property Law and ramifications for statutory interpretation». En Shyamkrishna Balganesh (editor), Intellectual property and the common law. Cambridge: Cambridge University Press.

Merges, Robert (2012). «Priority and novelty under the AIA». Berkeley Technology Law Journal, 27: 1.023-1.046. DOI: 10.2139/ssrn.2130209. 
Merges, Robert, Peter Menell y Mark A. Lemley (2012). Intellectual property in the new technological age. Nueva York: Aspen.

Merton, Robert (1977). La sociología de la ciencia 1: Investigaciones teóricas y empíricas. Madrid: Alianza.

Patel, Surendra J. (1974). «The patent system and the third world». World Development, 2 (9): 3-14. DOI: 10.1016/0305-750X(74)90084-9.

Prager, Frank (1944). «History of intellectual property: From 1545 to 1787». Journal of the Patent Office Society, 26 (11): 711-760.

-. (1946). «Brunelleschi's patent». Journal of the Patent \& Trademark Office Society, 28 (2): 109-135.

Pretorius, Willem (2002). «TRIPS and developing countries: How level is the playing field?». En Peter Drahos y Ruth Mayne (editores), Global intellectual property rights. Londres: Palgrave Macmillan.

Ricketson, Sam (2015). The Paris Convention for the protection of industrial property: A commentary. Oxford: Oxford University Press.

RoDRÍGUEZ, Enrique Barrero (2016). Hacia un nuevo régimen jurídico de la creación industrial. Madrid: Marcial Pons.

SÁIz, J. Patricio (1996). Legislación histórica sobre propiedad industrial: España (17591929). Madrid: Oficina Española de Patentes y Marcas.

SÁnCHEZ GARCía, Luz (2017). «Cómo preservar la novedad en la divulgación de las invenciones universitarias: el periodo de gracia». Diario La Ley, 9.033.

Smiers, Joost (2006). Un mundo sin copyright: Artes y medios en la globalización. Barcelona: Gedisa.

Stallman, Richard (2004). Software libre para una sociedad libre. Madrid: Traficantes de Sueños.

STIGLitz, Joseph (2007). «Knowledge as a global public good». En Global Public Goods: International Cooperation in the 21st Century (pp. 308-325). Oxford: Oxford University Press. DOI: 10.1093/0195130529.003.0015.

Wong, Sulan (2015). «Patents and scientific research: Five paradoxical scenarios». En Oswaldo Terán y José Aguilar (editores), Societal benefits of freely accessible technologies and knowledge resources. Hershey: IGI Global.

WonG, Sulan, Joan Ramos Toledano y Julio Rojas-Mora (2018). «Sobre la (in)compatibilidad de la ciencia abierta con la novedad como estándar de patentabilidad». Revista Jurídica, 15: 88-103. DOI: 10.17151/jurid.2018.15.2.6.

ZIMAN, John (2003). ¿Qué es la ciencia? Madrid: Cambridge University Press. 


\section{Sobre las autoras}

Sulan Wong es profesora asociada del Departamento de Ciencias Jurídicas de la Universidad de La Frontera, Chile. Obtuvo su título de abogada en la Universidad de Los Andes, Venezuela. Doctora en Derecho por la Universidad de La Coruña, España. Ha tenido estancias de investigación en la Universidad de Barcelona, España, en la Universidad de Aviñón, Francia, y el Institut National de Recherche en Informatique et en Automatique, Francia. Miembro del Centro de Investigación sobre Desafíos Internacionales de la Universidad de La Frontera. Su correo electrónico es sulan. wong@ufrontera.cl. (D) https://orcid.org/oooo-0003-1684-0285.

Michelle Salazar Mackenna es egresada de la carrera de Derecho de la Universidad Católica de Temuco, Chile. Ayudante de investigación del Centro de Investigación sobre Desafíos Internacionales de la Universidad de La Frontera, Chile. Su correo electrónico es michelle.salazarmackenna@gmail.com. (D) https://orcid. org/0000-0002-5012-1279. 


\title{
REVISTA CHILENA DE DERECHO Y TECNOLOGÍA
}

La Revista de Chilena de Derecho y Tecnología es una publicación académica semestral del Centro de Estudios en Derecho Informático de la Facultad de Derecho de la Universidad de Chile, que tiene por objeto difundir en la comunidad jurídica los elementos necesarios para analizar y comprender los alcances y efectos que el desarrollo tecnológico y cultural han producido en la sociedad, especialmente su impacto en la ciencia jurídica.

\author{
EDITOR GENERAL \\ Daniel Álvarez Valenzuela \\ (dalvarez@derecho.uchile.cl) \\ SITIO WEB \\ rchdt.uchile.cl \\ CORREO ELECTRÓNICO \\ rchdt@derecho.uchile.cl \\ LICENCIA DE ESTE ARTÍCULO \\ Creative Commons Atribución Compartir Igual 4.o Internacional
}

\begin{abstract}
La edición de textos, el diseño editorial
y la conversión a formatos electrónicos de este artículo

estuvieron a cargo de Tipográfica

(www.tipografica.io).
\end{abstract}

